PSYCHOMETRIKA-VOL. 29 , No. 2

JUNE, 1964

\title{
A GRAPH THEORETIC APPROACH TO SIMILARITY RELATIONS*
}

\author{
Frank Harary \\ UNIVERSITY OF MICHIGAN
}

The term "similarity relation" is used extensively in the psychological literature (see, e.g., Restle [9]). Sometimes it is called a "proximity relation," as in Coombs [2]. The underlying idea is always that there is a collection of objects, each of which has a real number value associated with it. There is also a fixed positive number $t$, for "threshold." Whenever the magnitude of the difference between the values of two objects is less than $t$, they are said to be similarly related to each other, or, more briefly, similar. Thus every object is necessarily similar to itself. Also it is obvious that if one object is similar to another object, then the second object must be similar to the first. But it is not always true that if among three distinct objects, the first is similar to the second and the second to the third, then the first must be similar to the third. For the difference in values between the first and third objects may exceej tine zinshold even though each of their values is within the threshold of the value of the second object. For these reasons, similarity relations have been described axiomatically as binary relations which are reflexive and symmetric, but not necessarily transitive. Logically, this third stipulation couldn't be more superfluous. For if a condition is not necessarily true, this is captured simply by not asserting that it is true. We shall see that similarity relations are naturally expressible as graphs and that their concepts are subsumed within the framework of graph theory. As an illustration of the psychological literature, we will refer to [3], where it is suggested correctly that symbolic logic is also an appropriate "mathematical model" for similarity relations.

\section{Goodman-Galanter Model}

In a recent paper, Galanter [3] developed "an axiomatic study of sensory order and measure," based on the work of Goodman [5], whence the "Goodman-Galanter Model" discussed in Coombs [2]. While the terminology and notation of symbolic logic is used in [3], the basic concepts of the treatment

*The preparation of this article was supported by Grant NSF-G-17771 from the National Science Foundation. 
are graph theoretic in nature. Graph theory has already been useful in providing a logical framework for several concepts in social psychology; see, for example, $[1,6,7]$. Our purpose is to demonstrate the appropriateness of graph theory as a model for similarity relations. We shall also see that the conceptual simplicity provided by this kind of geometric approach suggests certain natural directions for further research. We conclude by describing a particular problem in graph theory, solved very recently, which is precisely related with this aspect of psychophysics, namely, the characterization of "interval graphs."

In [3], experimental tests are performed on similarity of pairs of colors. In the corresponding abstract system, there is a collection of stimulus events $a, b, c, \cdots$ and a binary relation denoted by $M$ called "matching." The stimuli stand for colors and relation $M$ for indistinguishability between stimuli. The relation $M$ is postulated to be symmetric and reflexive but "not necessarily transitive." The central assumption is that two stimuli are to be regarded as equal if and only if the set of stimuli which match with the first is identical with the matching set of the second stimulus. The motivation for this logical approach is clearly stated in the following paragraph from [3].

To avoid philosophical issues which have no place in the discussion of the logical theory, we have restated Goodman's nominalistic definitions and axioms in terms of a finite Boolean algebra. This restatement identifies an element in the interpretation of the theory, say a "color," with the set or class of phenomenal appearances of a particular physical energy configuration. The elements of the system could just as easily be conceived as probability distributions of certain responses to the configurations. In that case, elements of the system would match if the difference between moments of the distributions did not reach some arbitrarily (perhaps physiologically) determined value. Considered as sets of stimuli, matching obtains in those cases in which, on some selected fraction of trials, pairs selected from the sets in question are not discriminable.

\section{Some Concepts from Graph Theory}

A graph consists of a finite collection of points $a, b, c, \cdots$ together with a prescribed subset of the set of all unordered pairs of distinct points. Each of these unordered pairs, $a b$, is a line of the graph. From the viewpoint of the theory of binary relations, a graph corresponds to an irreflexive symmetric relation on this set of points.

Fig. 1 depicts a graph $G$ of four points and four lines. The presence of line $a b$ indicates the existence of a specified symmetric relationship between the two entities $a$ and $b$. The points might represent people, and the lines some relationship such as mutual liking, or the points could stand for colors and the lines for indistinguishability, etc. With the popular interpretation, Fig. 1 indicates that mutual liking exists between those pairs of people $a, b, c$, and $d$ joined by lines. Thus $d$ is in the pleasant relation with all other persons, while $c$ is in the relation only with $d$. Fig. 1 could be used, 


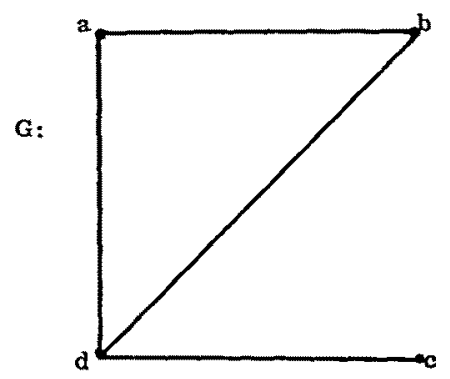

Figure 1

of course, to represent other kinds of relationships between varieties of entities.

\section{Binary Relations}

A binary relation $R$ between pairs of entities is reflexive if every entity is in the relation to itself. On the other hand, $R$ is irreflexive if no entity is in the relation to itself. The only (unimportant) difference between a graph as just defined and a similarity relation is that a graph is irreflexive while the similarity relation is necessarily reflexive.

For brevity, the notation $a R b$ is used to indicate that $a$ is in the relation $R$ to $b$. Thus $R$ is symmetric if whenever $a R b$, then $b R a$. Similarly, $R$ is transitive if for distinct entities $a, b$, and $c, a R b$ and $b R c$ together imply aRc. An equivalence relation is reflexive, symmetric, and transitive. Abstractly, equivalence relations play the role of an equal sign. Thus every equivalence relation is a similarity relation, but not conversely.

We now describe the generalization of a graph which corresponds to reflexive relations such as similarity. A loop in a graph is a line joining a point with itself. The r-graph of $G$ is obtained from a graph $G$ on adding a loop at every point (" $r$ " for reflexive). Fig. 2 shows the $r$-graph of Fig. 1.

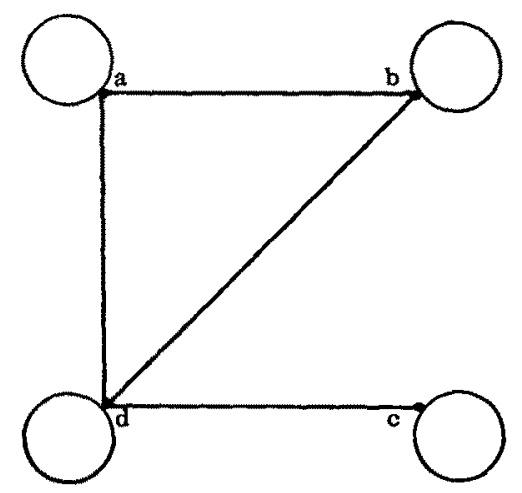

Fradre 2 
Of course, $r$-graphs and similarity relations are entirely coextensive, being merely different ways of expressing exactly the same properties.

\section{Betwixt and Beside}

Two points of a graph are adjacent if they are joined by a line. For any point $v$ in a given graph $G$, let $N(v)$, called the neighborhood of $v$, be the collection of all points of $G$ with which $v$ is adjacent. Using medieval terminology, Galanter [3] calls the neighborhood of a point its "manor." In the remainder of this section, his terminology will be indicated by quotation marks. When $G$ is an $r$-graph, it follows that every point is in its own neighborhood. Let us say that two points $u$ and $v$ of a graph are $N$ equivalent, denoted $u N v$, if their neighborhoods consist of exactly the same points, i.e., $N(u)=N(v)$. This is an equivalence relation, for it is reflexive, symmetric, and transitive, and expresses the condition for two stimuli to be regarded as "identical."

We use capital letters $A, B, C, \cdots$ for the neighborhoods of points $a, b, c, \cdots$, respectively. By a "matching graph" is meant an $r$-graph whose points represent stimuli and whose lines indicate indistinguishability. Additional conditions stipulated on the matching graph $M$ are that there are at least two points and there are no isolated points (i.e., each point is adjacent to at least one other point). We illustrate some of these concepts with the $r$-graph $M$ of Fig. 3. In this $r$-graph, it is understood that there is a loop at each point even though these are not drawn. The neighborhoods

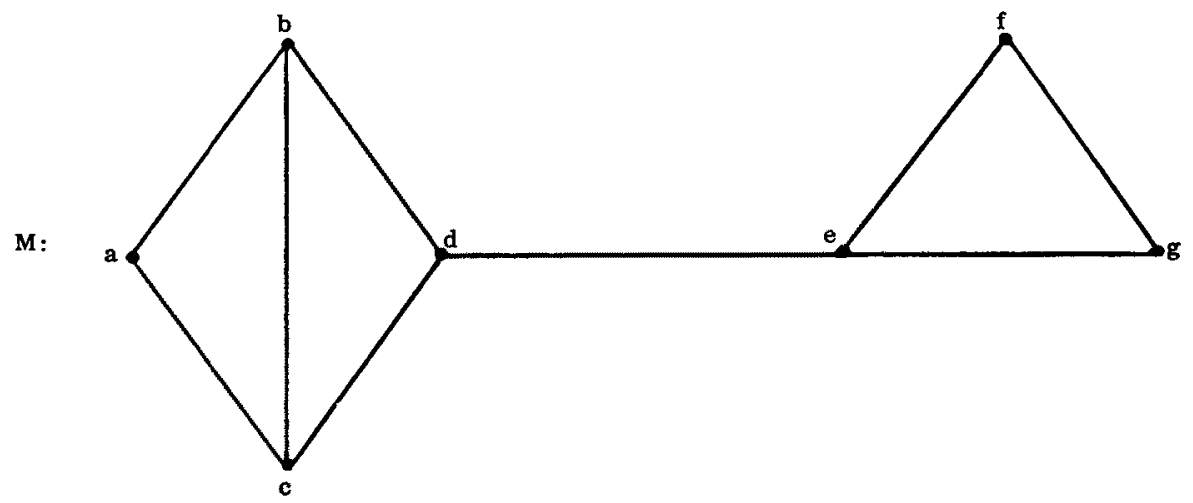

Frgure 3

of all the points of $M$ are given by

$$
\begin{aligned}
A & =\{a, b, c\}, \\
B=C & =\{a, b, c, d\},
\end{aligned}
$$




$$
\begin{aligned}
D & =\{b, c, d, e\}, \\
E & =\{d, e, f, g\}, \\
F=G & =\{e, f, g\} .
\end{aligned}
$$

Since $B=C$ and $F=G$, it follows that points $b$ and $c$ are $N$-equivalent, as also are points $f$ and $g$. If 'we denote the combined points by $b c$ and $f g$, then we get the graph $\bar{M}$ obtained from Fig. 3 on coalescing $N$-equivalent points, as shown in Fig. 4.

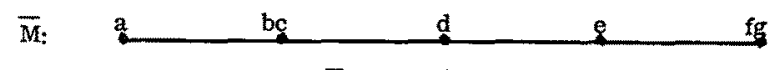

Figure 4

In order to state the definition of "betwixtness" of three points of $M$, we require some of the basic definitions of set theory. A set is a collection of elements. For any set $S$, let $|S|$ be the number of elements in $S$. The union of two sets $A$ and $B$, written $A \cup B$, is the set of all elements which lie in at least one of the two sets $A$ and $B$. Their intersection $A \cap B$ contains all elements which lie in both sets. The difference $A-B$ is the set of those elements which are in $A$ but not in $B$. Finally, their symmetric difference, $A \oplus B$, is defined by

$$
A \oplus B=(A-B) \cup(B-A),
$$

or in other symbols,

$$
A \oplus B=(A \cup B)-(A \cap B) .
$$

For any three points $a, b$, and $c$ of the matching graph $M$, the point $b$ is "betwixt" $a$ and $c$, denoted ( $a b c)$, if the following conditions obtain.

i. The points $a, b$, and $c$ are pairwise adjacent.

ii. $|A \oplus C|>|A \oplus B|$ and $|A \oplus C|>|B \oplus C|$.

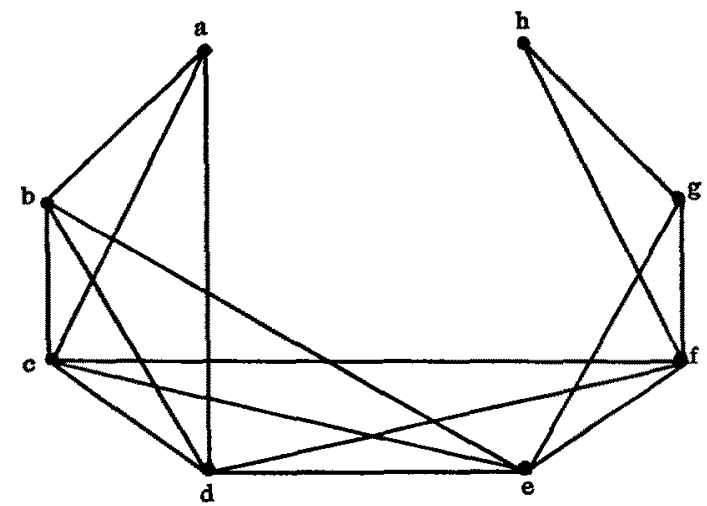

Fraure 5 
It is easy to verify that (cde) in the matching graph $M$ of Fig. 5. Both of the conditions of the definition of betwixtness are satisfied, namely,

i. $c d, c e$, and $d e$ are all lines of $M$;

ii. $|C \oplus E|=3,|D \oplus E|=2,|C \oplus D|=1$.

As immediate corollaries of the definition of betwixtness, it follows that

$(a b c)$ implies that the points $a, b, c$ are distinct;

$(a b c)$ implies $(c b a)$;

$(a b c)$ implies that neither of the betwixtness relationships $(a c b)$ or (bac) holds.

Another relation, denoted by $B$, is called "beside." Read $a B b$ as " $a$ is beside $b . "$ Then $a B b$ means that $a$ and $b$ are distinct matching points and there is no point betwixt them. Thus the relation $B$ is irreflexive and symmetric (but not necessarily transitive). It follows from the above definition that $B$ is an ordinary graph without any loops.

\section{Interval Graphs}

The traditional approach to psychophysics is to make a 1-to-1 correspondence between physical dimension and subjective experience, for example, between the wave length of light and the perceived color. The relation of betwixtness was developed in order to give a precise condition for one color to be between two others with respect to subjective perception. In structural terminology, this involves "linearizing" the points of a given graph in some natural order. However, it is known that there are graphs whose points cannot be linearized by any procedure whatsoever. An example is any graph consisting of a single cycle; a cycle of length 4 is shown in Fig. 6 .

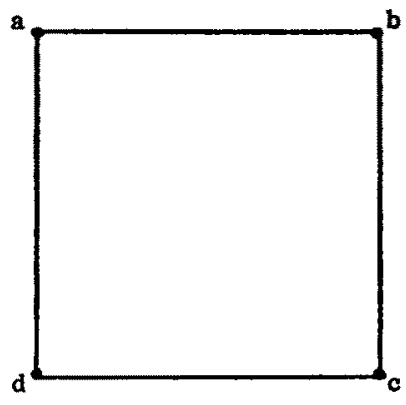

Frgure 6

There is an interesting area of graph theory which is related to this problem. It can be paraphrased as follows. Consider a collection $S$ of $n$ undergraduate students, all of whom know each other and have perfect 
recall. Each student $s_{i}$ visits the library exactly once in the afternoon, say from time $t_{i 0}$ to time $t_{i 1}$. At dinner, each student tells a graduate student in mathematics, named $X$, whom he saw in the library. The problem for $X$ is to determine the order in which $s_{1}, s_{2}, \cdots, s_{n}$ visited the library.

The time period during which $s_{i}$ was in the library can be represented by an interval on the $t$-axis from $t_{i 0}$ to $t_{i 1}$ as in Fig. 7 .

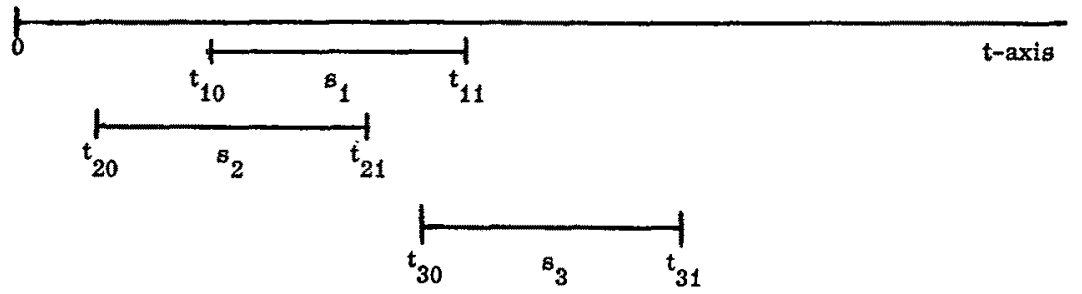

Frgure 7

Thus one student saw another in the library if and only if their intervals overlap. A graph can be drawn to contain this information by taking the points as students and the lines as mutual observation.

An interval graph is a graph whose points stand for intervals on the real axis, in which two points are adjacent if and only if their intervals overlap. It is clear that Fig. 6 is not an interval graph while Fig. 5 is one. In Fig. 8, we show a family of intervals whose interval graph is Fig. 5.

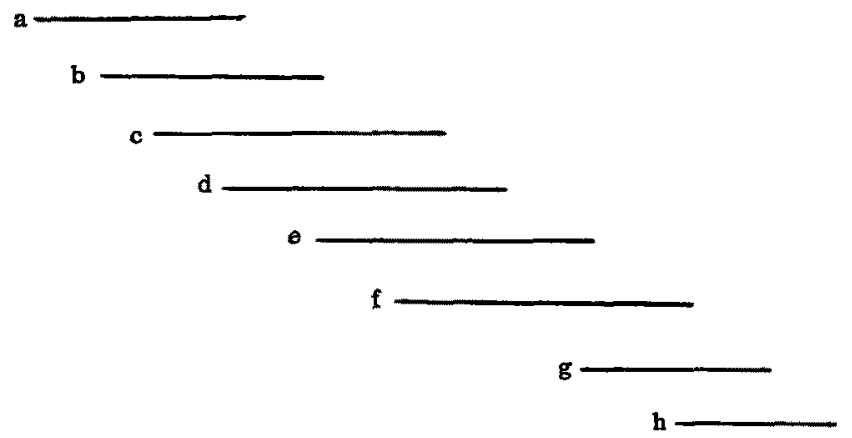

Fraure 8

It was until very recently an unsolved problem in graph theory, proposed by a Hungarian mathematician named Hajos, to characterize interval graphs. However, simultaneously and independently, solutions to this problem were found by Gilmore and Hoffman [4] and Lekkerkerker and Boland [8]. Because it is simpler to state, we will include here the criterion of [4]. A few additional definitions are required for this statement. 
The complement $\vec{G}$ of a given graph $G$ is that graph on the same set of points as $G$ in which two points are adjacent if and only if they are not adjacent in $G$. In Fig. 9, a graph $G$ and its complement $\vec{G}$ are shown.
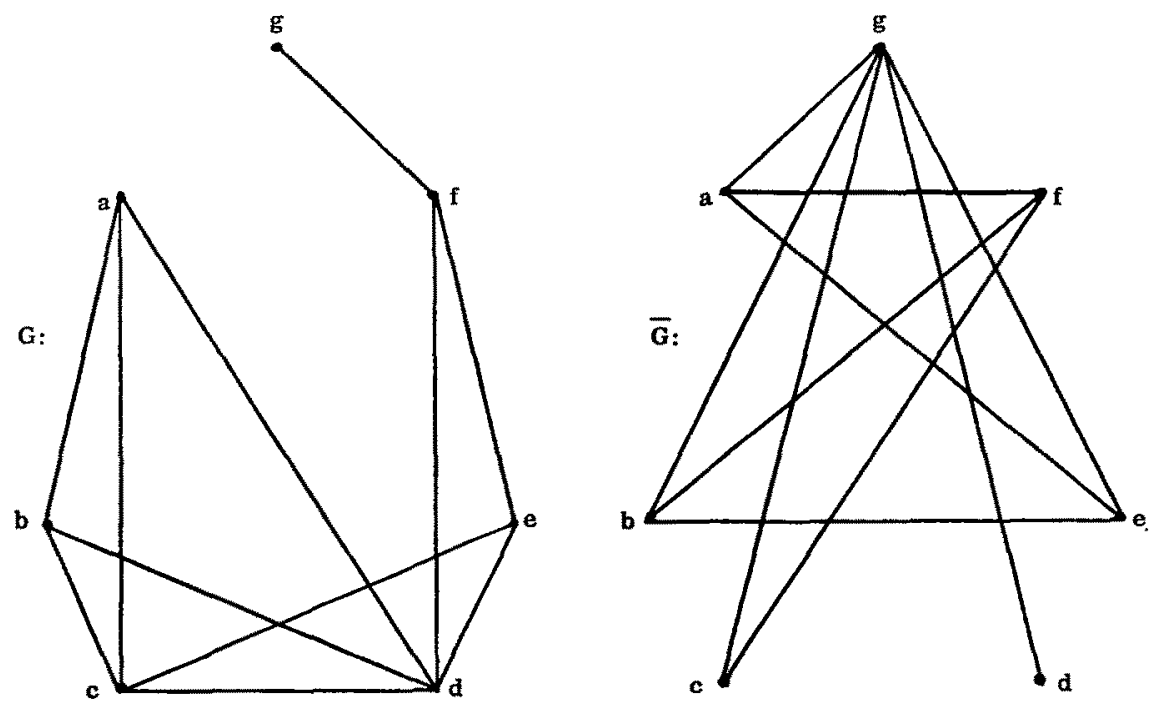

Figure 9

By a quadrilateral in $G$ is meant a cycle containing four lines. A diagonal of a quadrilateral $a b c d a$ is a line joining two nonconsecutive points, that is, line $a c$ or $b d$. An odd cycle of a graph has an odd number of lines. A triangular chord of a cycle of a graph is a line of the graph joining two points of the cycle which are separated by just one intermediate point of the cycle.

Theorem [4]. A graph $G$ is an interval graph if and only if every quadrilateral in $G$ has a diagonal and every odd cycle in $\bar{G}$ has a triangular chord.

This criterion is illustrated with the graph $G$ of Fig. 9 and its complement $\bar{G}$. The quadrilateral $a b c d a$ in $G$ has the lines $a c$ and $b d$ of $G$ as diagonals. The graph $\bar{G}$ has an odd cycle gafbeg, and this cycle has line $g b$ as a triangular chord.

The characterization given in [8] is more detailed in the sense that a specific list of configurations is provided, at least one of which must occur as part of any graph not an interval graph.

\section{Summary}

A similarity relation is reflexive and symmetric. A graph may be regarded as a geometric representation of an irreflexive symmetric relation; 
an $r$-graph then coincides with a similarity relation. For the realization of psychophysical phenomena, the points of a graph stand for the stimuli and a line for indistinguishability of a stimulus pair. Not all graphs, however, are likely to arise as similarity relations in a real situation. A special class of graphs, called interval graphs, does have this realistic property. These graphs are described and characterized.

\section{REFERENCES}

[1] Cartwright, D. and Harary, F. Structural balance: A generalization of Heider's theory. Psychol. Rev., 1956, 63, 277-293.

[2] Coombs, C. H. The theory of data. New York: Wiley, 1964.

[3] Galanter, E. H. An axiomatic and experimental study of sensory order and measure. Psychol. Rev., 1956, 63, 16-28.

[4] Gilmore, P. C. and Hoffman, A. J. A characterization of comparability graphs and of interval graphs. Unpublished report dated Feb. 15, 1962, IBM Research Center, Yorktown Heights, New York.

[5] Goodman, N. The structure of appearance. Cambridge, Mass.: Harvard Univ. Press, 1951.

[6] Harary, F. Graph theoretic methods in the management sciences, Mgmt Sci., 1959, 5, $387-403$.

[7] Harary, F., Norman, R. Z., and Cartwright, D. Structural models: An introduction to the theory of directed graphs. New York: Wiley, 1964, in press.

[8] Lekkerkerker, C. G. and Boland, J. C. Representation of a finite graph by a set of intervals on the real line. Fundamenta Mathematicae, 1962, 51, 45-64.

[9] Restle, F. A metric and an ordering on sets. Psychometrika, 1959, 24, 207-220.

Manuscript received $11 / 19 / 68$ 